\title{
Digestibilidad de forrajes arbóreos en bovinos utilizando jaulas metabólicas
}

\section{Aboreal forage digestibility in cattle using metabolic cages}

Roa $\mathrm{ML}^{1}$ y Céspedes $\mathrm{DA}^{2}$

${ }^{1}$ MSc. y ${ }^{2}$ Esp. Docentes Universidad de los Llanos, Escuela Ciencias Animales.

\section{mroa@unillanos.edu.co}

Recibido 3 de junio 2011 aceptado 12 de agosto 2011

\section{RESUMEN}

Para optimizar la producción animal en zonas tropicales, es necesario mejorar la calidad nutricional del ganado con forrajes que también ofrezcan mantenimiento nutritivo del suelo y las condiciones ambientales. El objetivo fue evaluar el potencial forrajero de cinco arbóreas adaptadas a la región de los llanos (Meta) mediante una prueba de digestibilidad in vivo en bovinos. Se utilizaron cinco animales con un peso promedio de $350 \pm 18,5 \mathrm{~kg}$, se estabularon en jaulas. Los tratamientos fueron: un testigo de pasto a voluntad (Braquiaria decumbens) (T0) cinco kg de matarratón (Gliricidia sepium) (T1); cinco kg de pízamo (Erythrina glauca) (T2); cinco kg de cayeno (Hibiscus rosa-sinensis) (T3); cinco kg de nacedero (Trichanthera gigantea) (T4) y cinco kg de poró (Erythrina poeppigiana) (T5). Se midió el consumo y excreción de heces y orina y también se calculó la energía digestible (ED), metabólica (EM), neta de mantenimiento $(E N m)$ y neta de producción (ENp). Las jaulas metabólicas constaban de comedero, bebedero, piso de malla para la recolección de heces, con ángulo de inclinación evitando el contacto con la orina. En el Laboratorio de Nutrición Animal se determinó materia seca (MS), proteína, grasa, fibra cruda (FC), cenizas, extracto no nitrogenado (ENN), fibra en detergente neutro (FDN) y fibra en detergente ácido (FDA) a los forrajes y excretas para determinar los coeficientes de digestibilidad (cod). El modelo experimental fue un diseño completamente al azar con diez repeticiones y 6 tratamientos, se aplicaron las pruebas de Tukey. Los cod de todos los nutrientes fueron inferiores en el tratamiento testigo $(P>0.05)$, lo mismo que los nutrientes digestibles totales (56.4\%). Cayeno y nacedero mostraron las mayores digestibilidades $(p>0.05)$, con relación a los demás tratamientos. T0 dispone de 
menos ( $p>0.05)$ ENp, $16,52 \%$, en comparación con cayeno (18,77\%) y nacedero $(18,21 \%)$. Las menores pérdidas por heces y orina e incremento calórico los presentaron cayeno y nacedero. Se concluye que la proteína y la energía se aprovechan en alto grado cuando se suplementa con éstas dos forrajeras, lo que demuestra su excelente calidad nutricional.

Palabras claves: Digestibilidad, energía, metabolismo.

\begin{abstract}
To optimize animal production in tropical areas, it is necessary to improve the nutritional quality forage. Besides to maintenance of soil and environmental conditions excellent. The objective was to evaluate the forage potential of five arboreal forages adapted to Meta department of Colombia by testing in vivo digestibility in cattle. Five steers were used with an average weight of $350 \pm 18.5$ $\mathrm{kg}$, were housed in cages. The treatments were: Grass (Brachiaria decumbens) (T0), five kg of Gliricidia sepium (T1), five kg of Erythrina glauca (T2), five $\mathrm{kg}$ of Hibiscus rosa-sinensis (T3), five $\mathrm{kg}$ of Trichanthera gigantea (T4) and five $\mathrm{kg}$ of Erythrina poeppigiana (T5). The food Consumption, excretion of feces and urine were measured. The digestible energy (DE), metabolic energy (ME), net energy of maintenance (NEm) and net energy of production (NEp) were also calculated. Metabolic cages had feeders, drinkers and mesh floor to collect feces, they had a tilt angle, avoiding contact with urine. In the Animal Nutrition Laboratory analyze to feed and feces: Dry matter (DM), protein, fat, crude fiber (CF), ash, nitrogen free extract (EFN), neutral detergent fiber (NDF) and acid detergent fiber (FDA) to determine digestibility coefficients (cod). The experimental model was a completely randomized design with ten replicates and 6 treatments were applied Tukey tests. The cod of all nutrients were lower in the T0 ( $P>0.05)$, as well as total digestible nutrients (56.4\%). $\mathrm{H}$ rosa-sinenesis and $T$ gigantea showed the highest digestibility $(p<0,05)$ compared to other treatments. T0 got less $(p>0.05)$ NEp $(16.52 \%)$, compared to $H$. rosa-sinenesis (18.77\%) $T$ gigantea (18.21\%). These two forages showed less losses in feces, urine and metabolic heat. It is concluded that protein
\end{abstract}


and energy are used to a high degree when supplemented with these two forage crops, which shows their excellent nutritional quality.

Keywords: Digestibility, energy, metabolism.

\section{INTRODUCCIÓN}

En el Pie de Monte y la Altillanura existen grandes extensiones de pastos nativos y mejorados que contienen baja cantidad de nutrientes debido a la limitación del suelo y a las dos estaciones climáticas (verano-invierno), lo cual ha ocasionado buscar sistemas alternativos para optimizar la producción, con esto se busca mejorar la calidad de las praderas tradicionales para que ofrezcan a su vez cambios positivos en el suelo, beneficiando las condiciones ambientales y por lo tanto la calidad de los forrajes y la producción animal (Perez y Perez, 2003). Para lograr buenas alternativas, existen en esta región árboles y arbustos nativos 0 introducidos, de los cuales se ha generado poca información referente a su potencialidad forrajera para el uso de la nutrición animal como parte integrante de una pradera (Rincón et al., 2002).

Para realizar la degradación de los forrajes el tracto digestivo de los bovinos cuenta con diversos microorganismos como bacterias, hongos y protozoos, que son los encargados de romper las partículas para facilitar el aprovechamiento de los nutrientes que finalmente va a utilizar el animal en la producción de carne o leche. Este proceso se encuentra influenciado por varios factores, los cuales son principalmente: Edad del animal, tipo de producción, calidad y cantidad de la fibra del forraje, nivel nutricional del animal, frecuencia de alimentación y uso de suplementos. (Van Lier y Regueiro, 2008).

Es así, que la digestión de la proteína depende de su solubilidad para que sea atacada por las bacterias proteolíticas ruminales, o sea utilizada directamente por el tracto posterior digestivo como proteína verdadera o sobrepasante que es la que genera la mayor cantidad de aminoácidos esenciales, los cuales son indispensables para la producción de carne o leche. Cuando se le suministra una dieta balanceada al bovino y el suficiente nitrógeno amoniacal a los 
microorganismos ruminales, estos no degradan la proteína de los alimentos, que es de alta calidad y por lo tanto puede ser aprovechada más eficientemente por el organismo del animal. (Villalobos, 2000; Galindo y Marrero, 2005).

La degradación de la fibra del forraje se realiza principalmente en el rumen por bacterias que producen la celulasa y que pertenece al género Ruminococcus Bacteroides y Butyrivibrio, esta digestión también se puede llevar a cabo en el intestino delgado y grueso, aunque en poca proporción, esto depende del tipo de dieta y forraje, tasa de pasaje, tamaño de partículas y otros factores. Además, la fibra del alimento tiene como función mantener cercano a la neutralidad el pH del rumen lo que favorece el incremento de la población de las bacterias celulolíticas y por tanto la disponibilidad de ácidos grasos volátiles que son la principal fuente de energía del rumiante (Yang et al., 2000; Bowman et al., 2003).

NRC 2000 y NRC 2001, establecen que uno de los métodos para determinar la digestibilidad y aprovechamiento de los alimentos en este caso forrajes, es el uso de la jaula metabólica para hacer mediciones de consumo y excreción y de esta manera calcular los coeficientes de digestibilidad de cada uno de los nutrientes que componen un alimento, estos son: proteína, grasa, fibra cruda y extracto no nitrogenado. Con la información se puede establecer el contenido energético y su distribución dentro del metabolismo del animal.

De acuerdo a estas investigaciones, el objetivo fue realizar pruebas de digestibilidad in vivo, así como de consumo, en seis bovinos estabulados en jaulas metabólicas, alimentados con una dieta testigo de pasto (Brachiaria decumbens) y suplementadas con cinco especies arbóreas forrajeras. También se calculó el contenido de: energía bruta (EB), nutrientes digestibles totales (NDT), energía digestible (ED), energía metabolizable (EM), energía neta de mantenimiento (ENm) y energía neta de producción (Enp).

\section{MATERIALES Y MÉTODOS}

La investigación se realizó en el municipio de Villavicencio, vereda Barcelona, en la granja y el laboratorio de Nutrición Animal de la Universidad de los Llanos. Esta 
zona tiene una altitud de 465 metros sobre el nivel del mar, una temperatura promedio de 27 grados centígrados y una precipitación anual entre 1900 a 2300 $\mathrm{mm}$.

Se utilizaron seis bovinos machos de un peso promedio de $350 \pm 18,5 \mathrm{~kg}$ alojados en jaulas metabólicas. El tiempo de duración del experimento fue de 25 días, con quince (15) días de adaptación a las dietas experimentales y diez (10) días de recolección de muestras. Al testigo (T0) se le suministró Brachiaria a voluntad únicamente y a los otros tratamientos, además del pasto, se suplementaron con cinco kilogramos de las siguientes especies forrajeras: T1: Matarratón (Gliricidia sepium), T2: Pízamo (Erythrina glauca), T3: Cayeno (Hibiscus rosa-sinensis), T4: Nacedero (Trichanthera gigantea) y T5: Poró (Erythrina poeppigiana). El ofrecimiento del pasto Brachiaria fue fresco cortado de los potreros de la granja y se suministró durante todo el día, las arbóreas se suministraban en las horas de la mañana (8:00 a.m.).

Las jaulas metabólicas constan de un comedero, bebedero de cisterna, piso de malla para facilitar la recolección de heces, cuyo ángulo de inclinación permite que el excremento ruede hacia el recipiente donde se recolectan, evitando su contacto con la orina. La recolección de heces se realizó en forma cada 24 horas a las 8:00 am, con el fin de realizar su peso diario. De estos excrementos se tomaban dos muestras aproximadamente de 300 gramos para ser analizadas en el laboratorio de Nutrición Animal donde se les determinó materia seca (MS), proteína, grasa, fibra cruda $(F C)$, cenizas, extracto no nitrogenado (ENN), fibra en detergente neutro (FDN) y fibra en detergente ácido (FDA) (Tabla 1). A los forrajes consumidos (Brachiaria y arbóreas) también se les realizó las mismas pruebas nutricionales con el fin de determinar su aprovechamiento (Tabla 2), utilizando la metodología AOAC, 2006.

Valorando el consumo y la excreción de los nutrientes se determinó el coeficiente de digestibilidad (cod) de todos los nutrientes aplicando las siguientes fórmulas (NRC, 2001 y NRC, 2002): 


$$
C o d=\frac{\text { Nutriente consumido }- \text { Nutriente excretado }}{\text { Nutriente consumido }}
$$

Con estos resultados se calculó los nutrientes digestibles totales (NDT)

$$
\% N D T=\% \text { Proteína } * \operatorname{cod}+\% F C * \operatorname{cod}+\% E N N * \operatorname{cod}+\% \operatorname{grasa} * \operatorname{cod} * 2.25
$$

EB $($ megacal $/ \mathrm{Kg} \mathrm{MS})=\%$ proteína $* 0.04+\% F C * 0.04+\% E N N * 0.04+\%$ grasa $* 0.09$

ED $($ megacal $/ \mathrm{Kg} \mathrm{MS})=\%$ NDT*0.04409

$\mathrm{EM}(\mathrm{megacal} / \mathrm{Kg} \mathrm{MS})=E D * 0.82$

ENm (megacal/Kg MS) $=0.029 \%$ NDT-0.29

Energía fecal (megacal/Kg MS) (EF) $=E B-E D$

Energía urinaria (megacal/Kg MS) $(\mathrm{EU})=E D-E M$

Incremento calórico (megacal/Kg MS) (IC) $=5 \%$ de $E M=E M^{*} 0.05$

Energía Neta (megacal $/ \mathrm{Kg} \mathrm{MS})(\mathrm{EN})=E M-I C$

ENp (megacal/Kg MS) (ENp) $=E N-E N m$

Tabla 1. Composición (\%) de las heces recolectadas de bovinos en jaulas metabólicas

\begin{tabular}{lcccccc}
\hline Nutrientes & T0 & T1 & T2 & T3 & T4 & T5 \\
\hline Materia seca & 22.3 & 25.0 & 23.0 & 40 & 41 & 32 \\
Proteína & 5.2 & 4.8 & 3.9 & 3.0 & 6.8 & 4.5 \\
Grasa & 2.2 & 1.6 & 2.4 & 2.7 & 3.1 & 3.6 \\
Fibra cruda & 25.4 & 24.8 & 31.2 & 19.8 & 20.4 & 11.0 \\
ENN & 51.4 & 50.0 & 48 & 51.2 & 47.9 & 58.7 \\
Cenizas & 6.8 & 10.8 & 6.5 & 10.3 & 9.8 & 9.2 \\
FDN & 39.5 & 47.2 & 40.2 & 57.0 & 62.1 & 59.8 \\
FDA & 35.8 & 39.2 & 38.6 & 40.8 & 40.1 & 47.8 \\
\hline
\end{tabular}

El modelo experimental empleado es un diseño completamente al azar con diez repeticiones y 6 tratamientos. Las variables evaluadas fueron: Consumo de forrajes, excreción por heces, digestibilidades: Materia seca (MS), proteína, grasa, extracto no nitrogenado (ENN), fibra cruda (FC), fibra detergente neutro (FDN) y fibra detergente ácido (FDA) y determinaciones de energía: EB, ED, EM, ENm, 
ENp, EF y EU. Se realizó un análisis de varianza, y haciendo una prueba de comparación múltiple Tukey, utilizando el SPSS versión 10.

Tabla 2. Análisis nutricional de los forrajes utilizados en el experimento

\begin{tabular}{ccccccc}
\hline Nutrientes (\%) & Brachiaria & Matarratón & Pízamo & Cayeno & Nacedero & Poró \\
\hline Materia Seca & 30.37 & 27.0 & 26.75 & 24.68 & 20.34 & 20.87 \\
Proteína & 7.15 & 20.6 & 17.96 & 13.91 & 19.23 & 21.23 \\
Grasa & 1.83 & 3.5 & 4.28 & 4.24 & 4.32 & 3.52 \\
Fibra Cruda & 19.16 & 11.8 & 27.97 & 6.93 & 2.76 & 7.63 \\
ENN & 58.76 & 53.2 & 37.76 & 58.13 & 50.14 & 51.0 \\
Cenizas & 6.68 & 6.1 & 3.93 & 7.85 & 16.54 & 8.12 \\
FDN & 53.92 & 49.0 & 51.09 & 35.8 & 42.75 & 47.53 \\
FDA & 30.0 & 21.1 & 38.51 & 13.73 & 27.4 & 39.66 \\
\hline
\end{tabular}

\section{RESULTADOS Y DISCUSIÓN}

El mayor porcentaje de proteína lo presentaron: poró, matarratón y nacedero. Arosemena, 2008 y Polo, 2008, indica que este nutriente es muy variable porque influye el clima, la época, corte y la edad, a medida que ésta aumenta, la proteína disminuir, sin embargo los valores obtenidos en el presente experimento fueron superiores a $19 \%$, mientras que los obtenidos por este autor no pasaron del $17 \%$, de lo cual se deduce que la zona donde se cultiva la especie es determinante, puesto que en el cayeno el contenido de proteína fue menor $(13,91 \%)$ comparado con el reportado por Urdaneta y colaboradores, 1997 en Venezuela (19.6\%).

Los consumos de materia seca fueron mayores en los tratamientos con matarratón, pízamo y cayeno en comparación con los demás tratamientos (Tabla 3). En el testigo se observó el menor consumo de MS, materia orgánica y proteína y el mayor consumo de FDN, lo cual indica que el Brachiaria tiene un alto contenido de componentes de la pared celular con relación a las demás especies arbóreas (Tabla 3). El tratamiento con pízamo, cayeno y nacedero presentaron los mayores consumos de MS y proteína. 
Los coeficientes de digestibilidad de todos los nutrientes fueron inferiores en el tratamiento testigo $(P>0.05)$, lo mismo que los nutrientes digestibles totales (56.4\%), se observó una alta digestibilidad de la proteína en todos los forrajes, de lo cual se puede deducir que este nutriente se aprovecha en alto grado, lo que demuestra una excelente calidad de este nutriente en los forrajes estudiados (Tabla 4). El tratamiento con cayeno presentó las mayores digestibilidades $(P>0.05)$, con relación a los demás tratamientos (Tabla 4). Es de anotar, Pérez et al., 2005, en trabajos con en el matarratón, determinaron que la manera como se seca la muestra afecta no sólo el contenido de proteína sino la digestibilidad de este nutriente, pues la muestra secada mediante microondas presenta valores más altos que con estufa a $60^{\circ} \mathrm{C}$ : proteína $(27,89 \%$ VS $17 \%$ y digestibilidad (65.1\% VS $47.6 \%$ ). Resultados que no concuerdan con los obtenidos en la presente investigación, puesto que las digestibilidades de este nutriente fueron superiores al $79 \%$ en todos los forrajes, cuyas muestras fueron secadas en estufa a $60^{\circ} \mathrm{C}$.

Tabla 3 Consumo diario de los diferentes nutrientes en bovinos en jaulas metabólicas (gramos)

\begin{tabular}{lcccccc}
\hline \multicolumn{1}{c}{ Nutrientes } & T0 & T1 & T2 & T3 & T4 & T5 \\
\hline Materia seca & 8432 & 8459 & 8500 & 8507 & 8486 & 8446 \\
Mat. Orgánica & 602,9 & 1742,6 & 1526,6 & 1183,3 & 1631,9 & 1793,0 \\
Proteína & 154,3 & 296,1 & 363,8 & 360,7 & 366,6 & 297,3 \\
Fibra cruda & 1615,6 & 998,2 & 2377,5 & 589,5 & 234,2 & 644,4 \\
ENN & 4954,6 & 4500,3 & 3209,6 & 4945,0 & 4255,1 & 4307,3 \\
Grasa & 563,3 & 516,0 & 334,1 & 667,8 & 1403,7 & 685,8 \\
FDN & 4546,5 & 4145,0 & 4342,7 & 3045,4 & 3627,9 & 4014,2 \\
FDA & 2529,6 & 1784,9 & 3273,4 & 1168,0 & 2325,3 & 3349,5 \\
\hline
\end{tabular}

En la distribución de energía en los seis tratamientos que fueron suministrados a los bovinos, la EB se unificó para todos los tratamientos en $3.57 \mathrm{mcal} / \mathrm{Kg}$ de MS y con base en este dato se hicieron los cálculos para realizar la distribución de energía (mcal/kg MS) de aprovechamiento y de pérdidas (Tabla 5, Figuras 1 a 5). 
Tabla 4. Digestibilidad (\%) y NDT de los Nutrientes de cada tratamiento con bovinos en jaulas metabólicas

\begin{tabular}{ccccccc}
\hline Parámetros & T0 & T1 & T2 & T3 & T4 & T5 \\
\hline Mat. seca & $60.0^{\mathrm{a}}$ & $67.5^{\mathrm{b}}$ & $63.8^{\mathrm{a}}$ & $75.0^{\mathrm{c}}$ & $70.0^{\mathrm{b}}$ & $68.0^{\mathrm{b}}$ \\
Proteína & $79.0^{\mathrm{a}}$ & $82.7^{\mathrm{a}}$ & $82.2^{\mathrm{a}}$ & $90.4^{\mathrm{b}}$ & $83,1^{\mathrm{b}}$ & $90.0^{\mathrm{b}}$ \\
Grasa & $60.8^{\mathrm{a}}$ & $62.2^{\mathrm{ab}}$ & $63.2^{\mathrm{ab}}$ & $66.1^{\mathrm{b}}$ & $64.8^{\mathrm{ab}}$ & $61.7^{\mathrm{ab}}$ \\
ENN & $65.2^{\mathrm{a}}$ & $85.1^{\mathrm{cd}}$ & $88.2^{\mathrm{cd}}$ & $90.2^{\mathrm{d}}$ & $83.4^{\mathrm{c}}$ & $75.0^{\mathrm{b}}$ \\
Fibra cruda & $51.0^{\mathrm{a}}$ & $63.2^{\mathrm{b}}$ & $63.0^{\mathrm{b}}$ & $75.0^{\mathrm{c}}$ & $60.0^{\mathrm{b}}$ & $65.0^{\mathrm{b}}$ \\
FDN & $54.0^{\mathrm{a}}$ & $62.0^{\mathrm{b}}$ & $63.2^{\mathrm{b}}$ & $68.0^{\mathrm{c}}$ & $64.0^{\mathrm{b}}$ & $63.0^{\mathrm{b}}$ \\
FDA & $47.0^{\mathrm{a}}$ & $59.0^{\mathrm{b}}$ & $57.0^{\mathrm{b}}$ & $61.0^{\mathrm{b}}$ & $57.0^{\mathrm{b}}$ & $59.0^{\mathrm{b}}$ \\
NDT & $56.4^{\mathrm{a}}$ & $64.5^{\mathrm{b}}$ & $59.8^{\mathrm{a}}$ & $70.5^{\mathrm{b}}$ & $67.6^{\mathrm{b}}$ & $66.3^{\mathrm{b}}$ \\
\hline
\end{tabular}

Letras distintas en la misma fila son diferentes $(p<0.05)$.

Tabla 5. Distribución de la energía de los tratamientos utilizado en bovinos (Megacalorías/kg MS)

\begin{tabular}{ccccccc}
\hline ÍTEM & T0 & T1 & T2 & T3 & T4 & T5 \\
\hline EB & 3.57 & 3.57 & 3.57 & 3.57 & 3.57 & 3.57 \\
ED & $2.49^{\mathrm{a}}$ & $2.84^{\mathrm{c}}$ & $2.64^{\mathrm{b}}$ & $3.11^{\mathrm{d}}$ & $2.98^{\mathrm{c}}$ & $2.92^{\mathrm{c}}$ \\
EF & $1.08^{\mathrm{d}}$ & $0.72^{\mathrm{c}}$ & $0.93^{\mathrm{d}}$ & $0.46^{\mathrm{a}}$ & $0.59^{\mathrm{b}}$ & $0.64^{\mathrm{b}}$ \\
EM & $2.04^{\mathrm{a}}$ & $2.33^{\mathrm{c}}$ & $2.16^{\mathrm{b}}$ & $2.55^{\mathrm{d}}$ & $2.44^{\mathrm{c}}$ & $2.40^{\mathrm{d}}$ \\
EU & $0.45^{\mathrm{a}}$ & $0.51^{\mathrm{b}}$ & $0.47^{\mathrm{a}}$ & $0.56^{\mathrm{b}}$ & $0.54^{\mathrm{b}}$ & $0.53^{\mathrm{b}}$ \\
IC & $0.10^{\mathrm{a}}$ & $0.12^{\mathrm{a}}$ & $0.11^{\mathrm{a}}$ & $0.13^{\mathrm{a}}$ & $0.12^{\mathrm{a}}$ & $0.12^{\mathrm{a}}$ \\
EN & $1.94^{\mathrm{a}}$ & $2.22^{\mathrm{c}}$ & $2.05^{\mathrm{b}}$ & $2.42^{\mathrm{d}}$ & $2.32^{\mathrm{c}}$ & $2.28^{\mathrm{c}}$ \\
ENm & $1.35^{\mathrm{a}}$ & $1.58^{\mathrm{b}}$ & $1.44^{\mathrm{a}}$ & $1.75^{\mathrm{c}}$ & $1.67^{\mathrm{bc}}$ & $1.63^{\mathrm{b}}$ \\
ENp & $0.59^{\mathrm{a}}$ & $0.63^{\mathrm{a}}$ & $0.61^{\mathrm{ab}}$ & $0.67^{\mathrm{b}}$ & $0.65^{\mathrm{b}}$ & $0.64^{\mathrm{ab}}$ \\
\hline
\end{tabular}

Letras distintas en la misma fila son diferentes $(p<0.05)$.

El testigo dispone de la menor cantidad $(p<0.05)$ de: ED (2.49), EM (2.04) y ENp (0.59) cifras inferiores en comparación con los demás tratamientos, los cuales aprovechan mejor el alimento en ED y EM, con la utilización de ENp cayeno y nacedero fueron superiores $(p<0.05)$. También se observa en el tratamiento con cayeno se presentó menos pérdidas totales $(p<0.05)$ sumando $E F$, EU e IC $(1.15$ mecal/ $\mathrm{kg}$ de MS), lo que equivale al $32,3 \%$ de la EB suministrada en el alimento. Se deduce que las mayores digestibilidades de sus nutrientes favorecen, la 
asimilación de la energía, disminuyendo la perdida de nutrientes por las excretas y nitrógeno por orina.
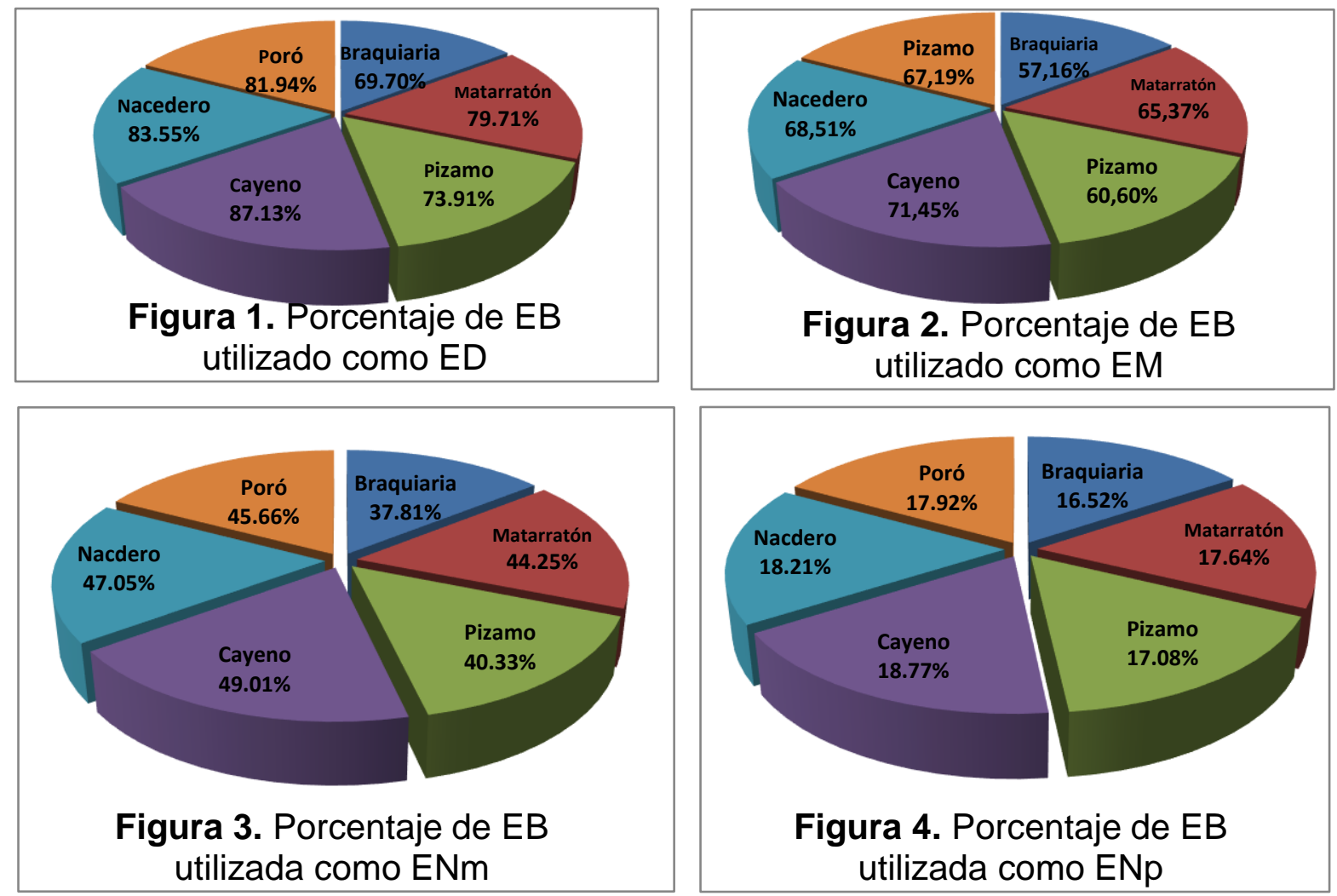

Figura 4. Porcentaje de EB utilizada como ENp

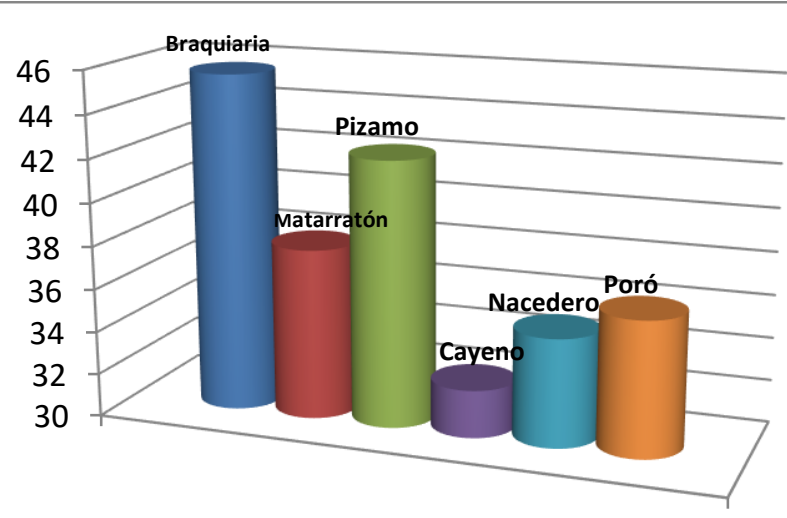

Figura 5. Porcentaje de pérdidas Totales: EF, EU, IC 


\section{CONCLUSIONES}

La proteína fue el nutriente que presentó el mayor coeficiente de digestibilidad en todos los tratamientos, las dietas con nacedero y cayeno mostraron las mayores digestibilidades en comparación con las demás. La eficiencia de utilización de la energía se incrementa cuando los animales reciben un suplemento arbóreo especialmente cuando se suministra nacedero o cayeno, se concluye que el Brachiaria decumbens es un pasto mejorado que puede ser utilizado en la alimentación de bovinos en alto grado, pero es necesario suplementar al ganado con un forraje más digestible y con mayor contenido de proteína y energía, con el fin de suplir adecuadamente los requerimientos nutricionales de los bovinos. También queda demostrado que los forrajes pueden utilizarse para complementar las gramíneas.

\section{BILIOGRAFİA}

1. Álvarez C. SPSS 10 (programas para computador) | estadística spss 10 para windows (programas para computador) |Análisis de datos [CD-Rom]. Madrid: Universidad Complutense. 2001.

2. AOAC. Official Methods of Analysis $\left(18^{\text {th }}\right)$ Association of Official Analytical Chemists, Arligton, VA. Washington, D.C. 2006.

3. Arosema, J. Efecto de la altura y épocas corte sobre la calidad y producción de la biomasa de nacedero (T. gigantea). Panamá, IDIAP. 2008.

4. Bowman GR, Beauchemin KA, Shelford JA. Fibrolytic enzymes and parity effects on feeding behavior, salivation, and ruminal $\mathrm{pH}$ of lactating dairy cows. J Dairy Sci. 2003. 86 (2): 565-575.

5. Galindo J, Marrero Y. Manipulación de la fermentación microbiana ruminal. Revista Cubana de Ciencia Agrícola. 2005. 39: 439-450.

6. Gómez E, Rodríguez L, Murgueitio E, Rios C, Molina C, Molina E, Molina P. Árboles y arbustos forrajeros utilizados en la alimentación animal como fuente proteica. CIPAV. Cali, Colombia. 2002: 146.

7. Jiménez G, López M, Nahed J, Ochoa S, De Jong G. Árboles y arbustos forrajeros de la región norte-tzotzil de Chiapas, México. Vet. Méx [online]. 2008: 39: (2) 199-213 [citado 2011-11-01], Disponible en: http://www.scielo.org.mx/scielo.php?script=sci arttext\&pid=S030150922008000200009\&lng=es\&nrm=iso

8. National Research Council (NRC). The Nutrient Requirements of Dairy Cattle, $7^{\text {th }}$ ed. Revised Edition. National Academy of Sciences, Washington, DC. 2001: 381. 
9. National Research Council (NRC). The Nutrient Requirements of beef Cattle, $7^{\text {th }}$ ed. Revised Edition. National Academy of Sciences, Washington, DC. 2000: 234.

10. Perez I, Perez B. Informe final proyecto: Evaluación agronómica y productiva de especies forrajeras en la Orinoquia Colombiana. CORPOICA. Cl La Libertad. Villavicencio. Meta. 2003:45.

11. Pérez AG, Abadía B, Arreaza L.C Aplicación De Una Metodología Para Cuantificar La Digestibilidad Intestinal Proteica En Rumiantes REV CORPOICA. 2005: 6:1:8.

12. Polo E. Preparando a los pequeños y medianos ganaderos para la competitividad; manejo de especies forrajeras arbustivas que incrementan beneficios en los sistemas doble propósito en Panamá. Panamá. PROMEGA. 2008: 32.

13. Rincón A, Cuesta P, Perez R, Bueno G, Pardo O, Gomez J. Manual técnico Producción y utilización de recursos forrajeros en sistemas de producción bovina de la Orinoquia y el Piedemonte Caqueteño. CORPOICAFEDEGANMADR. Santa fe de Bogotá. Colombia. 2002: 76.

14. Urdaneta M, Kaas, Rosero O, Parra N, Quintero A. Composición química y Digestibilidad de nuevas especies arbustivas utilizando dos métodos de secado. Rev Cient, FCV-LUZ. 1997: 7 (1): 17-22.

15. Van Lier E, Regueiro M. Digestión en retículo-rumen. Departamento de producción animal y pasturas. Universidad La República. Montevideo, Uruguay 2008: 30p.

16. Villalobos JC. Interrelación de suplementos proteicos y energéticos con la calidad de forraje de animales en pastoreo. VIII Congreso Internacional de Nutrición Animal. Chihuahua, México. 2000: 3-44.

17. Yang W.Z., Beauchemin K.A., Rode L.M. A comparison of methods of adding fibrolytic enzymes to lactating cow diets. J. Dairy Sci. 2000. 83: 2512-2520. 